\title{
Higher Blood Vascular Cell Adhesion Molecule-I is Related to the Increased Risk of Cardiovascular Events in Chronic Obstructive Pulmonary Disease
}

This article was published in the following Dove Press journal: International Journal of Chronic Obstructive Pulmonary Disease

\author{
Jinlin $\mathrm{Li}^{\prime}$ \\ Qi Wang' \\ Qingping Zhang ${ }^{2}$ \\ Zhengyan Wang' \\ Xin $\mathrm{Wan}^{3}$ \\ Chenfang Miao ${ }^{4}$ \\ Xi Zeng ${ }^{5}$
}

'Department of Respiratory Medicine, Suizhou Hospital, Hubei University of Medicine, Suizhou, Hubei 44I300, People's Republic of China; ${ }^{2}$ Department of Otolaryngology Head and Neck Surgery, Suizhou Hospital, Hubei University of Medicine, Suizhou, Hubei 44I300, People's Republic of China; ${ }^{3}$ Department of Critical Care Medicine, Mindong Hospital of Fujian Medical University, Fuan, Fujian 355000, People's Republic of China; ${ }^{4}$ Department of Anesthesiology, Mindong Hospital of Fujian Medical University, Fuan, Fujian 355000, People's Republic of China; ${ }^{5}$ Department of Geriatrics, Mindong Hospital of Fujian Medical University, Fuan, Fujian 355000, People's Republic of China
Correspondence: Xi Zeng Department of Geriatrics, Mindong Hospital of Fujian Medical University, No. 89 Heshan Road, Fuan City 355000, Fujian Province, People's Republic of China Tel +8618596658975

Email98729546।@qq.com
Background: Vascular cell adhesion molecule-1 (VCAM-1) is associated with vascularrelated inflammation and atherosclerosis. This study aimed to evaluate whether VCAM-1 can be used for an indication of increased risk of CV events in patients with COPD.

Methods: Serum VCAM-1 levels were measured in 163 COPD patients. All COPD patients were prospectively followed up for a median period of 48 months (range=3-54). Cox proportional hazard analysis was performed to evaluate the prognostic value of serum VCAM-1 for predicting CV events.

Results: Serum VCAM-1 levels were higher in COPD patients with CV events than in those without $\mathrm{CV}$ events $(1174.4 \pm 365.3 \mathrm{ng} / \mathrm{mL}$ vs $947.8 \pm 293.2 \mathrm{ng} / \mathrm{mL} ; P<0.001)$. The logistic regression analysis revealed that serum VCAM-1 (OR=1.750; 95\% CI, 1.324-2.428; $\left.P_{\text {trend }}=0.0012\right)$ was independently associated with CVD (cardiovascular disease) history after adjusting for age, sex, BMI, current smoker, current drinker, admission systolic and diastolic BP, LVEF and laboratory measurements in patients with COPD at baseline. The Kaplan-Meier analysis demonstrated that the rate of CV events was higher in COPD patients with serum VCAM-1 levels above the median $(517.3 \mathrm{ng} / \mathrm{mL})$ than in those with VCAM-1 levels below the median. The Cox proportional hazard analysis revealed that serum VCAM-1 (HR $=2.617$; 95\% CI, 1.673-5.328; $P_{\text {trend }}<0.001$ ) may be an independent prognostic factor for CV events in the COPD patients.

Conclusion: Our results suggested that serum VCAM-1 was significantly and independently associated with CV events in COPD patients. The inflammatory marker may help clinicians predict CV complications early.

Keywords: vascular cell adhesion molecule-1, chronic obstructive pulmonary disease, cardiovascular events, prognostic value

\section{Introduction}

Chronic obstructive pulmonary disease (COPD) is a smoking-related respiratory illness and is the fifth leading cause of disability and/or death worldwide. ${ }^{1,2}$ Patients with COPD are at a high risk of developing cardiovascular diseases (CVDs). ${ }^{3,4}$ Although the precise mechanisms are currently poorly understood, increased inflammation and oxidative stress in COPD are considered to be the main mechanisms causing an increased risk of a cardiovascular (CV) event. ${ }^{5}$ The early prediction of CVDs in patients with COPD might provide an opportunity to develop strategies to improve their prognosis.

Vascular cell adhesion molecule-1 (VCAM-1) is a member of the immunoglobulin (Ig) superfamily of proteins, which is composed of several extracellular Ig-like domains that contain disulfide-linked loops, a single type I transmembrane domain, 
and a 19 amino acid carboxyl-terminus cytoplasmic domain. $^{6-8}$ The expression of VCAM-1 is induced by other cytokines produced by neutrophils, macrophages and other inflammatory cells in vascular tissue. VCAM-1, in combination with other inflammatory factors, regulates immune surveillance and inflammatory responses. Novel serum biomarkers for the prevention of CVDs have been reported worldwide, but few studies have suggested that serum markers can improve risk prediction beyond classical risk factors such as high blood pressure and hyperlipidemia. VCAM-1 has been considered an early systemic inflammation marker and a risk marker for atherosclerosis-related diseases. ${ }^{9-12}$ Hence, high levels of this circulating molecule have been hypothesized to be associated with an increased risk of CVDs.

Importantly, existing studies have also suggested that COPD is associated with inflammatory responses and is considered to be a chronic inflammatory disease. ${ }^{13-16}$ Given the close association between VCAM-1 and inflammation, ${ }^{6-12}$ we speculated that VCAM-1 may be associated with CV events in COPD patients. This study aimed to assess whether increased serum VCAM-1 levels contribute to a higher risk for $\mathrm{CV}$ events, independent of clinically confounding factors in COPD patients. This is the first exploration of the prognostic value of VCAM-1 for predicting $\mathrm{CV}$ events in COPD patients.

\section{Materials and Methods Study Population}

We enrolled 163 COPD patients hospitalized because of COPD in an acute stage from the National Population Health Science Data Center in China between January 2014 and December 2015. This database contains most of the clinical data about COPD from many hospitals in China. Thus, this study has sufficient data for the analysis of the association of serum VCAM-1 with CV events in patients with COPD.

All COPD patients were stable for 6 months without acute diseases before admission. After standardized hospital treatment, all included patients with COPD were prospectively followed up after discharge. COPD was diagnosed by two respiratory specialists according to the same diagnostic criteria for all of these patients: "The Global Initiative for Chronic Obstructive Lung Disease". 17

Among the COPD patients, 46 had a CVD history. For the purpose of this study, a CVD history was defined as coronary heart disease, stroke and hypertension. Five patients with COPD were excluded from the study because of neoplastic diseases, chronic kidney disease, severe liver or lung diseases, and other severe systemic diseases.

Data on clinical characteristics, including age, sex, body mass index (BMI), current smoking status, current alcohol consumption status, blood pressure, and left ventricular ejection fraction (LVEF), were collected from patient interviews or medical records.

In addition, we also collected 102 healthy subjects as the control group, which was compared with the COPD patients for serum VCAM-1 levels. There were no differences in age or sex between the healthy subjects and the COPD patients, and the healthy subjects did not have any serious health problems. According to the Declaration of Helsinki guidelines, the Ethics Committee from Suizhou hospital of Hubei medical university approved this study, and all patients gave written informed consent.

\section{Follow-Up}

All patients with COPD were followed up for a median of 48 months (range $=3-54$ ) prospectively. Patients were followed up by review of their medical record or telephone 4 times a year until the occurrence of a $\mathrm{CV}$ event (the end point). The $\mathrm{CV}$ events in this study were myocardial infarction, heart failure (HF), stroke and CVD death. CVD death was defined as death due to myocardial infarction, progressive HF and stroke confirmed by two cardiologists.

\section{Measurement of VCAM-I}

Fasting venous blood samples were drawn from the COPD patients and healthy subjects within the first 24 hours after admission. The samples were prepared immediately by centrifugation and processed for the determination of VCAM-1 levels. At the commencement of the study, serum VCAM-1 was measured using commercially available ELISA kits according to the manufacturers' instructions (Abcam, UK). All samples were measured in duplicate. The mean of two measurements was taken (coefficient of variation of precision $[\mathrm{CV}],<5 \%$; detection limit, $5 \mathrm{ng} / \mathrm{mL}$ ).

\section{Laboratory Measurements}

The venous blood samples drawn from the COPD patients in the early morning within the first 24 hours after admission were also tested for hemoglobin $(\mathrm{Hb}), \mathrm{HbA} 1 \mathrm{c}$ (glycosylated hemoglobin), LDL (low-density lipoprotein), hs-CRP (hs-C reactive protein) and albumin (ALB) with the use of the Siemens ADVIA 2400 automatic biochemistry analyzer (Siemens AG). Serum N-terminal 
prohormone of B-type natriuretic peptide (NT-proBNP) levels were tested by using immunoassays on an ELECSYS2010 instrument (ELECSYS proBNP, Roche Diagnostics, Germany). The Modification of Diet in Renal Disease (MDRD) formula was used to calculate the estimated glomerular filtration rate (eGFR). ${ }^{18}$

\section{Statistical Analyses}

All of the analyses were conducted by using SPSS 23.0. The data that were not normally distributed are presented as the median (interquartile range) and were further analyzed by using the Mann-Whitney U-test. Normally distributed data are expressed as the mean \pm standard deviation (SD) and were further analyzed by independent $t$-test analysis Categorical variables were tested by using the chi-square test. In multivariable analyses, serum VCAM-1 levels were categorized by quartiles (quartile 4: $\geq 75$ th percentile, quartile $3: \geq 50$ th to 75 th percentile, quartile $2: \geq 25$ th to 50 th percentile, quartile $1:<25$ th percentile). At baseline, the independent relationship between serum VCAM-1 levels and CVD history in COPD patients was analyzed by multivariate logistic regression analysis. Cox proportional hazard analysis was used to assess whether serum VCAM-1 could be regarded as a valuable prognostic factor for $\mathrm{CV}$ events in COPD patients. $\mathrm{CV}$ event-free curves were constructed according to the Kaplan-Meier method and were compared using the Log rank test. $P \leq 0.05$ was considered to be statistically significant.

\section{Results}

\section{Clinical Characteristics at Baseline}

In order to compare the levels of serum VCAM-1 between COPD patients and healthy subjects, 102 age- and sexmatched subjects who had undergone a health examination were selected as the control group (Table 1). The patients with COPD had higher serum VCAM-1 levels (1034.6 \pm 345.1 $\mathrm{ng} / \mathrm{mL})$ than the control group $(733.2 \pm 226.5 \mathrm{ng} / \mathrm{mL})$. The clinical characteristics of the patients with COPD are presented in Table 2. CV events were identified in 41 (25.2\%) patients with COPD. According to the occurrence of $\mathrm{CV}$ events, 163 patients with COPD were divided into 2 groups. Patients with $\mathrm{CV}$ events tended to be current drinkers, older and have a higher BMI than patients without $\mathrm{CV}$ events (all $P<0.05)$. Patients with CV events had a lower LVEF, eGFR, $\mathrm{Hb}$ and ALB levels and higher VCAM-1, NT-proBNP, LDL, and hs-CRP levels than those without CV events (all
Table I Baseline Characteristics of Patients with COPD and Control Subjects

\begin{tabular}{|l|l|l|l|}
\hline Variables & $\begin{array}{l}\text { Healthy Control } \\
\text { Subjects } \\
(\mathbf{n}=102)\end{array}$ & $\begin{array}{l}\text { Patients with } \\
\text { COPD } \\
(\mathbf{n}=163)\end{array}$ & P value \\
\hline Age (years) & $64.8(60.1-72.1)$ & $65.2(60.9-72.6)$ & 0.753 \\
\hline $\begin{array}{l}\text { Sex (male/ } \\
\text { female) }\end{array}$ & $48 / 54$ & $77 / 86$ & 0.844 \\
\hline $\begin{array}{l}\text { VCAM-I } \\
\text { (ng/mL) }\end{array}$ & $733.2 \pm 226.5$ & $1034.6 \pm 345.1$ & $<0.001$ \\
\hline
\end{tabular}

Notes: Data are presented as mean \pm SD for normally distributed data and as median (interquartile range) for nonnormally distributed data.

Abbreviations: COPD, chronic obstructive pulmonary disease; VCAM-I, vascular cell adhesion molecule-I.

$P<0.001)$. There were no significant differences in the other variables, including sex and current smoker (all $P>0.05$ ).

Serum VCAM-I Levels Were Associated with CVD History in 163 COPD Patients at Baseline by Using Multivariate Logistic Regression Analysis

To assess the association of serum VCAM-1 with CVD history in COPD patients, multivariate logistic regression models were performed (Table 3). Model 1 indicated that increased serum VCAM-1 levels were significantly associated with a higher possibility of a CVD history after adjustments were made for age and sex. By adjusting for age, sex, BMI, current smoker, current drinker, admission systolic and diastolic BP, LVEF and treatments, the results of Model 2 were similar to those of Model 1. This association remained statistically significant and changed minimally after adding laboratory measurements to Model 2 (Model 3). The fully adjusted OR of CVD history in Model 3 was 1.750 (1.324-2.428) in quartile 4 (the highest) versus quartile 1 (the lowest) of serum VCAM-1 levels.

\section{Serum VCAM-I Levels Were Associated with CV Events in 163 COPD Patients During the Follow-Up of 4 Years by Using Cox Proportional Hazard Analysis}

All included COPD patients $(\mathrm{N}=163)$ were followed up for a median period of 48 months (range $=3-54$ ) prospectively. $\mathrm{CV}$ events occurred in 41 of the included patients. Among these, 15 patients died, and 13 of those deaths were caused by CVDs, which included myocardial infarction $(\mathrm{n}=6)$, stroke $(n=4)$ and progressive HF $(n=3)$. Additionally, 28 
Table 2 Clinical Characteristics Among 163 Patients with COPD at Baseline

\begin{tabular}{|c|c|c|c|c|}
\hline Variables & $\begin{array}{l}\text { All Patients } \\
(n=163)\end{array}$ & COPD without CV Events $(n=122)$ & $\begin{array}{l}\text { COPD with CV Events } \\
(n=4 I)\end{array}$ & $P$ value \\
\hline Age (years) & $65.2(60.9-72.6)$ & $63.4(59.2-70.1)$ & $66.3(63.5-76.9)$ & 0.017 \\
\hline Sex (men/women) & $77 / 86$ & $57 / 122$ & $20 / 41$ & 0.158 \\
\hline BMI & $26.2(23.4-27.3)$ & $25.6(22.5-27.7)$ & $27.6(24.6-28.9)$ & 0.013 \\
\hline Current smoker, n (\%) & $26(16.0)$ & $20(16.4)$ & $6(14.6)$ & 0.206 \\
\hline Current drinker, n (\%) & $57(35.0)$ & $38(3 I .1)$ & $19(46.3)$ & 0.009 \\
\hline Admission systolic BP $(\mathrm{mmHg})$ & $\mid 27.9(|| 2.5-\mid 46.8)$ & I26.1 (106.9-|45.5) & I28.5 (| |3.6-|49.2) & 0.113 \\
\hline Admission diastolic BP $(\mathrm{mmHg})$ & $78.7(71.2-86.5)$ & $77.5(66.3-80.2)$ & $79.6(76.5-88.3)$ & 0.327 \\
\hline LVEF, n (\%) & $63.1 \pm 4.3$ & $65.7 \pm 4.9$ & $60.2 \pm 4.1$ & $<0.001$ \\
\hline \multicolumn{5}{|l|}{ CVD history } \\
\hline Hypertension, n (\%) & $33(20.2)$ & $24(19.7)$ & $9(22.0)$ & 0.371 \\
\hline Diabetes mellitus, n (\%) & $48(29.4)$ & $35(28.7)$ & $13(31.7)$ & 0.295 \\
\hline Coronary heart disease, $\mathrm{n}(\%)$ & $33(20.2)$ & $18(14.8)$ & $15(36.6)$ & 0.002 \\
\hline Stroke, n (\%) & $23(14.1)$ & $10(8.2)$ & $13(31.7)$ & $<0.001$ \\
\hline Others, n (\%) & $12(7.4)$ & $8(6.6)$ & $4(9.8)$ & 0.150 \\
\hline \multicolumn{5}{|l|}{ Treatments } \\
\hline ACEI/ARB & $20 / 163$ & $14 / 122$ & $7 / 41$ & 0.004 \\
\hline beta-Blocker & $39 / 163$ & $29 / 122$ & $|0 / 4|$ & 0.310 \\
\hline $\mathrm{CCB}$ & $28 / 163$ & $21 / 122$ & $7 / 41$ & 0.779 \\
\hline Diuretic & $42 / 163$ & $32 / 122$ & $|0 / 4|$ & 0.413 \\
\hline Lipid-lowering drug & $24 / 163$ & $18 / 122$ & $6 / 41$ & 0.272 \\
\hline \multicolumn{5}{|l|}{ Laboratory measurements } \\
\hline eGFR $\left(\mathrm{mL} / \mathrm{min} / 1.73 \mathrm{~m}^{2}\right)$ & $56.8(45.1-63.7)$ & $63.1(57.1-66.2)$ & $52.7(43.2-60.5)$ & $<0.001$ \\
\hline NT-proBNP (pg/mL) & $623(428-78 I)$ & $437(277-670)$ & $688(52 \mid-947)$ & $<0.001$ \\
\hline $\mathrm{Hs}-\mathrm{CRP}(\mathrm{pg} / \mathrm{mL})$ & $7.3(2.6-13.5)$ & $5.7(2.1-8.4)$ & $9.8(3.7-17.6)$ & $<0.001$ \\
\hline $\mathrm{Hb}(\mathrm{g} / \mathrm{L})$ & $105(91-119)$ & $113(98-126)$ & $101(85-112)$ & $<0.001$ \\
\hline ALB $(g / L)$ & $38.1(36.6-4 \mid .2)$ & $39.2(37.4-44.0)$ & $36.3(34.2-39.3)$ & $<0.001$ \\
\hline HbAlc (\%) & $5.8(5.6-6.2)$ & $5.8(5.5-6.0)$ & $6.1(5.8-6.5)$ & 0.088 \\
\hline LDL (mmol/L) & $2.47 \pm 0.97$ & $2.16 \pm 0.89$ & $2.67 \pm 1.38$ & $<0.001$ \\
\hline VCAM-I (ng/mL) & $1034.6 \pm 345.1$ & $947.8 \pm 293.2$ & $1174.4 \pm 365.3$ & $<0.001$ \\
\hline
\end{tabular}

Notes: Data are presented as mean \pm SD for normally distributed data, as median (interquartile range) for nonnormally distributed data, and as $\mathrm{n}$ (\%) for categoric variables. Abbreviations: COPD, chronic obstructive pulmonary disease; CV, cardiovascular; BMI, body mass index; BP, blood pressure; LVEF, left ventricular ejection fraction; CVD, cardiovascular disease; ACEl, angiotensin-converting enzyme inhibitor; ARB, angiotensin receptor blocker; CCB, calcium channel blocker; eGFR, estimated glomerular filtration rate; NT-proBNP, N-terminal prohormone of B-type natriuretic peptide; Hb, hemoglobin; hs-CRP, hs-C reactive protein; ALB, albumin; HbAlc, glycosylated hemoglobin; LDL, low-density lipoprotein; VCAM-I, vascular cell adhesion molecule-I.

COPD patients had CV events that required rehospitalization due to myocardial infarction, stroke and progressive HF during the follow-up of 4 years.

Kaplan-Meier analysis demonstrated that COPD patients with serum VCAM-1 levels above the median $(517.3 \mathrm{ng} / \mathrm{mL})$ had a significantly higher rate of $\mathrm{CV}$ events than patients with serum VCAM-1 levels below the median value (Log rank test, $P<0.001$ ) (Figure 1). To further determine the independent association of serum VCAM-1 with $\mathrm{CV}$ events in patients with COPD, multivariate Cox proportional hazard regression analysis was used (Table 4). Multivariate Cox proportional hazard analysis suggested that VCAM-1 $\left(\mathrm{HR}=2.617 ; 95 \%\right.$ CI $\left.1.673-5.328 ; P_{\text {tend }}<0.001\right)$ was an independent prognostic factor for $\mathrm{CV}$ events after adjustments were made for age, sex, BMI, current smoker, current drinker, admission systolic and diastolic BP, LVEF, CVD history, treatments and laboratory measurements.

We performed an additional sensitivity analysis to evaluate the association of serum VCAM-1 with CV events in COPD patients who had no previous CVD history ( $\mathrm{n}=131)$. In these COPD patients, the number of total CV events was 27. Similarly, compared with COPD patients who had VCAM-1 levels below the median value, Kaplan-Meier survival curves showed that the rate of CV events was still higher in patients with VCAM-1 levels above the median (Log rank test, $P<0.001$ ) (data not 
Table 3 The Association Between the CVD History and VCAM-I in 163 Patients with COPD by Logistic Regression Analysis

\begin{tabular}{|l|l|l|l|}
\hline Variables & Model I & Model 2 & Model 3 \\
\hline VCAM-I levels (ng/mL) & & & \\
Quartile I & 1.000 (ref.) & 1.000 (ref.) & 1.000 (ref.) \\
Quartile 2 & $1.314(1.120-1.689)$ & $1.245(1.116-1.608)$ & $1.215(1.112-1.493)$ \\
Quartile 3 & $1.476(1.235-1.924)$ & $1.415(1.220-1.855)$ & $1.345(1.183-1.751)$ \\
Quartile 4 & $1.964(1.463-3.459)$ & $1.841(1.396-2.995)$ & $1.750(1.324-2.428)$ \\
P-trend & $<0.001$ & 0.001 & 0.012 \\
\hline
\end{tabular}

Notes: Model I: Adjusted for age and gender, Model 2: Adjusted for age, gender, BMI, current smoker, current drinker, admission systolic and diastolic BP, LVEF and treatments, Model 3: Adjusted for age, gender, BMI, current smoker, current drinker, admission systolic and diastolic BP, LVEF, treatments and laboratory measurements. Abbreviations: CVD, cardiovascular disease; COPD, chronic obstructive pulmonary disease; VCAM-I, vascular cell adhesion molecule-I; BMI, body mass index; BP, blood pressure; LVEF, left ventricular ejection fraction.

shown). The multivariate Cox analysis showed that higher VCAM-1 levels were still independently associated with a higher risk of $\mathrm{CV}$ events (data not shown).

\section{Discussion}

These are the new findings in this study: 1) COPD patients with CV events had higher serum VCAM-1 levels than those without $\mathrm{CV}$ events; 2) serum VCAM-1 was independently associated with a CVD history in COPD patients by multivariate logistic regression analysis; 3) VCAM-1 may be a valuable factor for predicting $\mathrm{CV}$ events by multivariate Cox proportional hazard analysis; and 4) KaplanMeier analysis suggested that COPD patients with higher VCAM-1 levels had significantly higher rates of $\mathrm{CV}$ events.

Patients with COPD show accelerated lung and vascular aging that may be the result of the activation of the inflammatory response and oxidative stress. ${ }^{19,20} \mathrm{COPD}$ significantly increases the risk of atherosclerosis-related

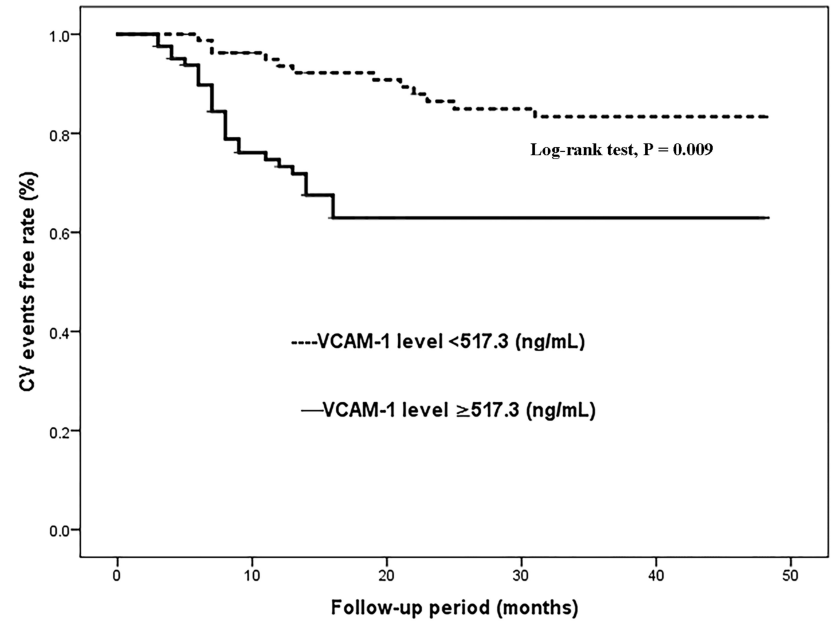

Figure I Kaplan-Meier analysis of cardiovascular (CV) events free stratified into 2 groups by the median levels of serum vascular cell adhesion molecule-I (VCAM-I). diseases, including myocardial infarction, stroke, carotid and other atherosclerosis-related diseases. ${ }^{21,22}$ Damage to the cerebral microvascular by atherosclerosis can result in both mild cognitive impairment or dementia that is significantly correlated with COPD severity. ${ }^{23-25}$ Around 9\% of COPD patients have peripheral vascular diseases, and these patients have reduced vascular function or adverse health status. ${ }^{26}$ Therefore, it is of great clinical significance to determine the independent risk factors or predictors of $\mathrm{CV}$ events in COPD patients.

VCAM-1 is composed of several extracellular Ig-like domains, and it plays an important role in immunity and inflammatory responses. ${ }^{6-8}$ As a member of the immunoglobulin superfamily, VCAM-1 is a cell surface protein and it is expressed by several cell types, including endothelial cells, macrophages, smooth muscles, and dendritic cells. ${ }^{27}$ Increased circulating VCAM-1 is a result of an excess surface expression or increased shedding, and it is a key player in the adhesion and transmigration of leukocytes from the circulation into the surrounding tissue and thus contributes to tissue inflammation. Some previous clinical studies have suggested that VCAM-1 is relevant to various cardiac diseases, including congestive heart failure, ischemic heart disease and rheumatic heart disease. ${ }^{28-31}$ Higher serum VCAM-1 levels are also significantly associated with newonset atrial fibrillation. ${ }^{32,33}$ Several basic studies have demonstrated that VCAM-1 expression is upregulated in the vascular tissue and it potentially contributes to inflammation-related pathological changes in vitro and in vivo. ${ }^{34,35}$

Our study population included patients who had been hospitalized for COPD, and the aim was to evaluate the value of serum VCAM-1 levels for predicting CV events in patients with COPD. In this study, we found that serum VCAM-1 had a close association with a CVD history at baseline, which may be partly or mostly explained by the mechanistic research performed in previous studies. ${ }^{6-8,34,35}$ 
Table 4 Cox Proportional Hazard Analysis for Predicting CV Events in 163 Patients with COPD

\begin{tabular}{|l|l|l|l|}
\hline Variables & Model I & Model 2 & Model 3 \\
\hline VCAM-I levels (ng/mL) & & & \\
Quartile I & 1.000 (ref.) & 1.000 (ref.) & 1.000 (ref.) \\
Quartile 2 & $2.011(1.400-3.69 I)$ & $1.865(1.313-3.207)$ & $1.594(1.210-3.088)$ \\
Quartile 3 & $2.460(1.744-4.249)$ & $2.217(1.523-4.014)$ & $2.154(1.456-3.449)$ \\
Quartile 4 & $3.152(2.093-6.150)$ & $2.846(1.774-5.871)$ & $2.617(1.673-5.328)$ \\
P-trend & $<0.001$ & $<0.001$ & $<0.001$ \\
\hline
\end{tabular}

Notes: Model I: Adjusted for age and gender, Model 2: Adjusted for age, gender, BMI, current smoker, current drinker, admission systolic and diastolic BP, LVEF, CVD history and treatments, Model 3: Adjusted for age, gender, BMI, current smoker, current drinker, admission systolic and diastolic BP, LVEF, CVD history, treatments and laboratory measurements.

Abbreviations: CVD, cardiovascular disease; COPD, chronic obstructive pulmonary disease; VCAM-I, vascular cell adhesion molecule-I; BMI, body mass index; BP, blood pressure; LVEF, left ventricular ejection fraction.

These studies have demonstrated that chronic vascular inflammation caused by COPD promotes atherosclerosisrelated CV events. The increased VCAM-1 levels may be the result of the aggravation of inflammation in COPD patients and it is involved in the occurrence and development of CVDs. The chronic inflammation may be a possible explanation for the increased VCAM-1 levels, which is consistent with our hypothesis and results. The early detection of CVDs in these patients may provide an opportunity to develop strategies aiming to reduce its medical burden and improve their prognosis. Our results seem to imply that serum VCAM-1 might be a sensitive biomarker for the early recognition of $\mathrm{CV}$ events in COPD patients.

This study has some strengths. We found that serum VCAM-1 may be used as a valuable prognostic marker for $\mathrm{CV}$ events in patients with COPD. The serum test is convenient and simple for application to COPD patients. We also ensured a comprehensive follow-up and rigorous adjudication of $\mathrm{CV}$ events. Finally, the VCAM-1 assay chosen for this analysis is both widely available and analytically stable.

\section{Limitations}

This study has several limitations. First, the study included a small sample size. Large-scale prospective cohort studies and randomized controlled trials are needed to better understand the association between serum VCAM- 1 and CV events in patients with COPD. Second, some inflammatory factors associated with VCAM-1 should also be evaluated in order to identify their prognostic value for predicting CV events. Third, confounding factors for COPD, such as treatment and respiratory function, were not included in this study, which may bring some biases to our results. Additionally, VCAM-1 was analyzed only once at enrollment, whereas the COPD patients were followed-up for 48 months. Thus, we are not able to exclude that the circulating VCAM-1 levels had changed substantially during the follow-up period. Furthermore, the patients were included in this study during a COPD exacerbation and we cannot exclude that this condition has not strongly affected the study outcomes.

\section{Conclusions}

Serum VCAM-1 is an independent prognostic factor for predicting $\mathrm{CV}$ events in patients with COPD. VCAM-1 might enable the early recognition of COPD patients at risk of developing $\mathrm{CV}$ complications. Future studies should address whether VCAM-1 is capable of improving the risk classification of $\mathrm{CV}$ events among COPD patients.

\section{Disclosure}

The authors report no conflicts of interest in this work.

\section{References}

1. Yang IA, Brown JL, Johnson G, et al. COPD-X Australian and New Zealand guidelines for the diagnosis and management of chronic obstructive pulmonary disease: 2017 update. Medical J Australia. 2017;207(10):436-442. doi:10.5694/mja17.00686

2. Vogelmeier CF, Criner GJ, Martinez FJ, et al. Global Strategy for the Diagnosis, Management, and Prevention of Chronic Obstructive Lung Disease 2017 Report. GOLD Executive Summary. Am J Respir Crit Care Med. 2017;195(5):557-582.

3. Leitao FF, Sin DD. COPD and cardiovascular diseases: now is the time for action. Thorax. 2018;73(9):799-800. doi:10.1136/thoraxjnl-2018-211553

4. Schneider C, Bothner U, S S J, et al. Chronic obstructive pulmonary disease and the risk of cardiovascular diseases. Eur J Epidemiol. 2010;25(4):253-260. doi:10.1007/s10654-010-9435-7

5. Barnes PJ. Senescence in COPD and its Comorbidities. Annu Rev Physiol. 2017;79(1):517. doi:10.1146/annurev-physiol-022516-034314

6. Kumar AG, Dai XY, Kozak CA, et al. Murine VCAM-1. Molecular cloning, mapping, and analysis of a truncated form. J Immunol. 1994;153:4088-4098.

7. Osborn L, Hession C, Tizard R, et al. Direct expression cloning of vascular cell adhesion molecule 1, a cytokine-induced endothelial protein that binds to lymphocytes. Cell. 1989;59:1203-1211. doi:10.1016/0092-8674(89)90775-7 
8. Willeit K, Pechlaner R, Willeit P, et al. Association Between Vascular Cell Adhesion Molecule 1 and Atrial Fibrillation. JAMA Cardiol. 2017;2(5):516-523.

9. Kaufmann BA, Sanders JM, Davis C, et al. Molecular imaging of inflammation in atherosclerosis with targeted ultrasound detection of vascular cell adhesion molecule-1. Circulation. 2007;116 (3):276-284. doi:10.1161/CIRCULATIONAHA.106.684738

10. Nankang H, Kevin, et al. N0X2 in lung inflammation: quantum dot based in situ imaging of NOX2-mediated expression of vascular cell adhesion molecule-1. Am J Physiol. 2014.

11. Cho YS, Kim CH, Ha TS, et al. Inhibition of STAT3 phosphorylation by sulforaphane reduces adhesion molecule expression in vascular endothelial cell. Can J Physiol Pharmacol. 2016;94(11):1. doi:10.1139/cjpp-2015-0150

12. Lemos JAD, Hennekens CH, Ridker PM. Plasma concentration of soluble vascular cell adhesion molecule-1 and subsequent cardiovascular risk. J Am Coll Cardiol. 2000;36(2):423-426. doi:10.1016/ S0735-1097(00)00742-7

13. Papi A, Bellettato CM, Braccioni F, et al. Infections and airway inflammation in chronic obstructive pulmonary disease severe exacerbations. Am J Respir Crit Care Med. 2006;173 (10):1114-1121. doi:10.1164/rccm.200506-859OC

14. Leena G, Christopher, et al. Eosinophilic airway inflammation: role in asthma and chronic obstructive pulmonary disease. Ther Adv Chronic Dis. 2016.

15. Gibbons M, Sethi T. Chronic obstructive pulmonary disease and lung cancer: inflammation, the missing link. Therapy. 2009;6(6):805-820. doi: $10.2217 /$ thy. 09.77

16. Wilkinson TMA, Donaldson GC, Johnston SL, et al. Respiratory syncytial virus, airway inflammation, and FEV 1 decline in patients with chronic obstructive pulmonary disease. Am J Respir Crit Care Med. 2006;173(8):871-876.

17. Antuni JD, Barnes PJ. Evaluation of individuals at risk for COPD: beyond the scope of the global initiative for chronic obstructive lung disease. Chronic Obstr Pulm Dis. 2016;3(3):653-667.

18. Levey AS, Stevens LA, Schmid $\mathrm{CH}$, et al. A new equation to estimate glomerular filtration rate. Ann Intern Med. 2009;150 (9):604-612.

19. Roversi S, Fabbri LM, D D S, et al. Chronic obstructive pulmonary disease and cardiac diseases: an urgent need for integrated care. $\mathrm{Am}$ J Respir Crit Care Med. 2016;rccm.201604-0690SO.

20. Wang LY, Zhu YN, Cui JJ, et al. Subclinical atherosclerosis risk markers in patients with chronic obstructive pulmonary disease: A systematic review and meta-analysis. Respir Med. 2017;123:18-27. doi:10.1016/j. rmed.2016.12.004

21. Zagaceta J, Bastarrika G, Zulueta JJ, et al. Prospective comparison of non-invasive risk markers of major cardiovascular events in COPD patients. Respir Res. 2017;18(1):175. doi:10.1186/s12931017-0658-y
22. Turgay S, Daniel RS, Jennifer N, et al. Epicardial fat, cardiovascular risk factors and calcifications in patients with chronic kidney disease. Clin Kidney $J$.

23. Lahousse L, Tiemeier H, Ikram MA, et al. Chronic obstructive pulmonary disease and cerebrovascular disease: A comprehensive review. Respir Med. 2015;S0954611115300299.

24. FitzGerald J, Mark. Risk of cardiovascular comorbidity in patients with chronic obstructive pulmonary disease: a systematic review and meta-analysis. Lancet Respir Med. 2015.

25. Morgan AD, Zakeri R, Quint JK. Defining the relationship between COPD and CVD: what are the implications for clinical practice? Ther Adv Respir Dis. 2018;12:175346581775052. doi:10.1177/ 1753465817750524

26. Sarah H-W, Rudolf, et al. Peripheral artery disease and its clinical relevance in patients with chronic obstructive pulmonary disease in the COPD and systemic consequences-comorbidities network study. Am J Respir Crit Care Med. 2017.

27. Hope SA, Meredith IT. Cellular adhesion molecules and cardiovascular disease; part I: their expression and role in atherogenesis. Intern Med J. 2003;33(8):380-386.

28. Blankenberg S, Barbaux S, Tiret L. Adhesion molecules and atherosclerosis. Atherosclerosis. 2003;170(2):191-203. doi:10.1016/ S0021-9150(03)00097-2

29. Chen MC, Chang HW, Juang SS, et al. Percutaneous transluminal mitral valvuloplasty reduces circulating vascular cell adhesion molecule-1in rheumatic mitral stenosis. Chest. 2004;125 (4):1213-1217. doi:10.1378/chest.125.4.1213

30. Malik I, Danesh J, Whincup P, et al. Soluble adhesion molecules and prediction of coronary heart disease: a prospective study and meta-analysis. Lancet. 2001;358(9286):971-976. doi:10.1016/ S0140-6736(01)06104-9

31. Tsutamoto T, Wada A, Maeda K, et al. AngiotensinII type1 receptor antagonist decreases plasma levels of tumor necrosis factor alpha, interleukin- 6 and soluble adhesion molecules in patients with chronic heart failure. J Am Coll Cardiol. 2000;35(3):714-721. doi:10.1016/ S0735-1097(99)00594-X

32. Willeit K, Pechlaner R, Willeit P, et al. Association between vascular cell adhesion molecule 1 and atrial fibrillation. JAMA Cardiol. 2017;2:516. doi:10.1001/jamacardio.2017.0064

33. Hugo V. Systemic vascular cell adhesion molecule-1 predicts the occurrence of post-operative atrial fibrillation. Int J Cardiol. 2011.

34. Goette A, Bukowska A, Lendeckel U, et al. AngiotensinII receptor blockade reduces tachycardia-induced atrial adhesion molecule expression. Circulation. 2008;117(6):732-742. doi:10.1161/ CIRCULATIONAHA.107.730101

35. Hammwöhner M, Ittenson A, Dierkes J, et al. Platelet expression of CD40/CD40 ligand and its relation to inflammatory markers and adhesion molecules in patients with atrial fibrillation. ExpBiol Med. 2007;232(4):581-589.

\section{Publish your work in this journal}

The International Journal of COPD is an international, peer-reviewed journal of therapeutics and pharmacology focusing on concise rapid reporting of clinical studies and reviews in COPD. Special focus is given to the pathophysiological processes underlying the disease, intervention programs, patient focused education, and self management protocols. This journal is indexed on PubMed Central, MedLine and CAS. The manuscript management system is completely online and includes a very quick and fair peer-review system, which is all easy to use. Visit http://www.dovepress.com/testimonials.php to read real quotes from published authors. 